\title{
Objective Monitoring of Brace Wearing Time in Adolescents with Scheuermann's Kyphosis
}

\author{
Pouya Sharifi ${ }^{1}$, Mojtaba Kamyab ${ }^{1}$, Taher Babaee ${ }^{1}$, Mohammad Saleh Ganjavian ${ }^{2}$ \\ ${ }^{I}$ Department of Orthotics and Prosthetics, School of Rehabilitation Sciences, Iran University of Medical Sciences, Tehran, Iran \\ ${ }^{2}$ Department of Orthopaedic Surgery, Shafa Yahyaiian Hospital, Iran University of Medical Sciences, Tehran, Iran
}

\section{Study Design: This was a prospective cohort study.}

Purpose: This study aimed to evaluate the potential differences between the objective and subjective bracing compliances of adolescents with Scheuermann's kyphosis.

Overview of Literature: Bracing is a well-documented intervention for managing adolescents with progressive thoracic Scheuermann's kyphosis, and the brace should be worn 23 hours every day. Most research studies that have investigated the efficacy of bracing have assumed that the patients wore the braces as advised or that the bracing time was measured subjectively. This may be one of the reasons for the conflicting reports regarding the efficacy of bracing.

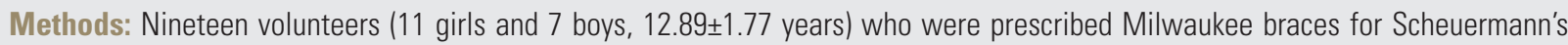
kyphosis were enrolled. Each brace was equipped with a miniature temperature logger to record the actual brace wearing time over a period of 3 weeks. The patients and their families were unaware of the mounted sensor. Each participants and/or parent was provided with a questionnaire to record the number of hours for which the brace was worn each day. In addition, the therapist asked each patient and/or his/her parent about the average number of hours that the brace was worn.

Results: The compliance rates measured using the temperature logger (16.00 \pm 4.90 hours daily) were significantly lower than those reported in the questionnaires $(19.52 \pm 6.04$ hours daily, $p<0.001)$ and the verbal responses $(20.21 \pm 6.05$ hours daily, $p<0.001)$. Moreover, there was no correlation of age, sex, and body mass index with brace compliance.

Conclusions: The braces were worn less often than reported by the patients and/or their parents. Therefore, objective compliance assessments of adolescents with Scheuermann's kyphosis in a brace are recommended for future studies.

Keywords: Spinal deformity; Hyperkyphosis; Scheuermann's kyphosis; Scoliosis; Brace compliance

\section{Introduction}

According to the Scoliosis Research Society, the normal range of thoracic kyphosis is $20^{\circ}-45^{\circ}$ in standing posteroanterior radiographs of the spine based on the Cobb angle [1]. Hyperkyphosis is a condition defined by in- creased posterior angulation of the thoracic spine (from T5 to T12) with a curve $>45^{\circ}$. Hyperkyphosis can appear because of several reasons, such as spinal fractures, neuromuscular diseases, Scheuermann's disease, and various unknown mechanisms.

One of the most common causes of hyperkyphosis is

Received Jan 12, 2019; Revised Apr 8, 2019; Accepted Apr 9, 2019

Corresponding author: Mojtaba Kamyab

Department of Orthotics and Prosthetics, School of Rehabilitation Sciences, Iran University of Medical Sciences, Madadkaran Ave., Shahnazari St., Madar Sq., Mirdamad Blvd., Tehran, Iran

Tel: +98-21-2222-0947 (Ext. 255), Fax: +98-21-2222-0946, E-mail: kamyab.m@iums.ac.ir 
Scheuermann's disease that affects $0.4 \%-10 \%$ of adolescents aged 10-16 years [2,3]. A skeletally immature patient with a progressive deformity requires active treatment, such as bracing and exercises, to control curve progression. Bracing is the most effective nonsurgical treatment for adolescents with flexible kyphotic deformities, and its effectiveness has been proven in several studies [46]. For patients with a thoracic apex at T8 or above, the Milwaukee brace is the most effective treatment for applying 3-point corrective forces to the apical vertebra $[4,6,7]$. Several factors, such as the age, sex, curve magnitude, and joint flexibility, contribute to the success of the orthotic treatment of spinal deformities $[8,9]$. However, the issue of brace compliance is another important factor [10-12]. If the patient does not follow the recommended brace wearing schedule (i.e., noncompliance), the treatment may not be successful.

Conventionally, brace compliance is evaluated using subjective methods, such as a patient diary, questioning the patient's parents or nurse, clinical evidence, and brace appearance $[13,14]$. However, these subjective methods are inaccurate, with a bias toward overestimation, because they are dependent on the patients or parents' estimates [15]. However, direct monitoring of brace compliance using several different sensors can guarantee higher precision with regard to the brace wearing time [11,16-18]. Despite the effectiveness of the Milwaukee brace for the treatment of Scheuermann's kyphosis, its compliance rate has not yet been evaluated. Moreover, when considering the differences between objective and subjective methods, the disparity could have great clinical importance. In recent years, a simple miniature temperature logger (DS1922L iButton; Maxim Integrated Products Inc., Sunnyvale, CA, USA) has been used to accurately monitor the brace wearing time [19].

Based on the above information, the present study aimed to objectively measure the Milwaukee brace compliance of adolescents with Scheuermann's kyphosis. We hypothesized that the brace-wearing times reported by the patients and their parents would differ significantly in the objective measurements.

\section{Materials and Methods}

\section{Participants}

This prospective cohort study was conducted on Scheuer- mann's kyphosis patients who were undergoing Milwaukee brace treatments. These braces were designed and manufactured for each patient individually. From June 2016 to October 2016, all the participants undergoing regular check-ups at the same spinal deformities clinic were requested to participate in this study. Total 21 participants were recruited.

The inclusion criteria were as follows: Scheuermann's kyphosis with prescription of braces, at least 10 years old at the beginning of bracing treatment, a Risser sign $\leq 2$, no previous treatment, and a sagittal Cobb angle of $45^{\circ}-74^{\circ}$. In addition, those with Cobb angles $>74^{\circ}$ who refused surgery were enrolled. The exclusion criteria were as follows: unwillingness for clinical referral for routine followup, missing sensor data, and an incomplete diary. All the participants who fulfilled the inclusion and exclusion criteria signed consent forms. This study was approved by the Human Research Ethics Committee of the Iran University of Medical Sciences (letter no., 1395.931367002).

\section{Instruments}

1) Objective compliance measurement

In the current study, the compliance was measured objectively using a temperature logger thermal-time sensor [19]. This small data logger $(17 \mathrm{~mm} \times 6 \mathrm{~mm})$ can accurately measure and record the temperature and time at variable intervals. This sensor comprises a waterproof stainless steel capsule that encases an $8-\mathrm{kB}$ data log memory, a digital thermometer, a real-time clock, and a battery. It has an integrated impact-resistant structure with no accessory parts. By setting the temperature logger to record data every 15 minutes, it is possible to record data for up to 85 days [19]. Given the wide potential applications of temperature loggers, they minimize the number of times that a patient needs to return to download the recorded information and release the memory. All the above-mentioned features, along with the affordable price, made this sensor an ideal choice for the researchers. The temperature logger was connected to a computer containing OneWireViewer software (Maxim Integrated Products Inc.) via its specific adapter that includes the ability to program the device based on the desired setting.

\section{2) Subjective compliance measurement}

Each patient's compliance was measured subjectively using a questionnaire designed for this purpose. The ques- 
tionnaire structure included a timetable that the patient and their parents could use to record the time duration for which the brace was worn each day [20]. In order to ensure patient collaboration, the patient's family was reminded via daily text message (short message service message). The patient's compliance, defined as his or her adherence to a prescribed wear schedule, was calculated by determining the ratio of hours for which the orthosis was worn to the hours of prescribed wear, multiplied by 100 to express the quotient as a percentage.

\section{Procedure}

The brace wearing time was assessed for at least 3 weeks using the following three methods: the questionnaire, the sensor, and a verbal report. Previous studies have shown that a measurement period of 2 weeks is sufficient to evaluate the usage pattern of braces [17].

In this study, the sensor was embedded in the kyphosis pad of the Milwaukee brace with 2-mm foam between the sensor and the patient's skin (Fig. 1). When the patient returned to the clinic for the bracing follow-ups, the sensor was removed from the brace, and the information was downloaded. The data were recorded as an algorithm that was ready for analysis (Fig. 2). The analysis of the recorded data was based on the following rules: (1) The accepted indoor temperature range was $>18^{\circ} \mathrm{C}$ and $<30^{\circ} \mathrm{C}$ (average, $\left.24^{\circ} \mathrm{C}-25^{\circ} \mathrm{C}\right)$. This temperature is defined as the thermal comfort zone in the field of meteorology. In the present study, the indoor temperature was measured using a sensor in three different environments for 3 days. The results of this experiment showed that the indoor temperature was $24^{\circ} \mathrm{C}-26^{\circ} \mathrm{C}$, with a threshold of $30^{\circ} \mathrm{C}$ [21]. This study was performed during the summer, and according to the Iran Meteorological Organization, the temperature of this geographic region overlapped with the sensor threshold temperature while it was recording the data. (2) In order
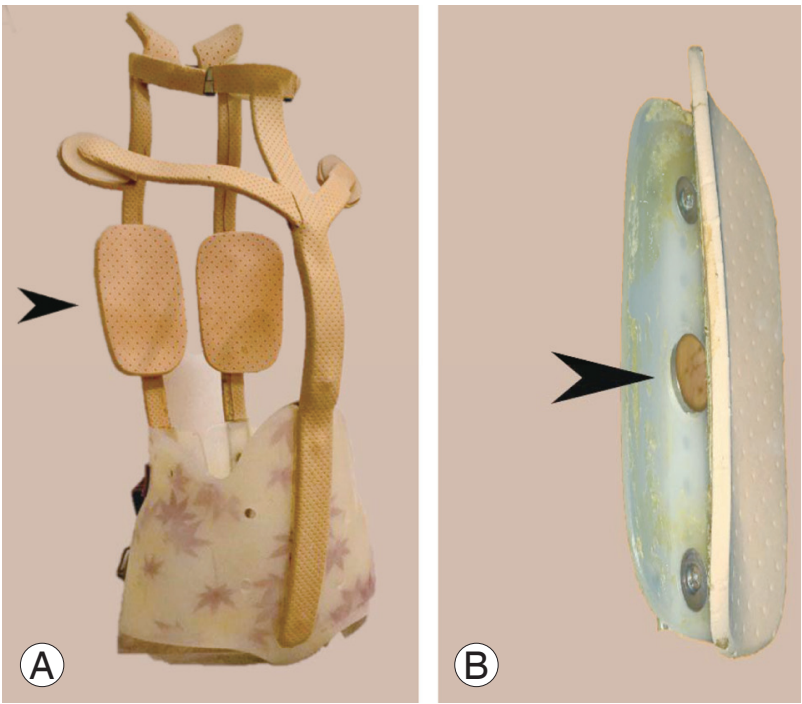

Fig. 1. (A) The Milwaukee brace for hyperkyphosis (arrowhead). (B) Placement of the Thermochron data logger on the thoracic pad of the brace (arrowhead).

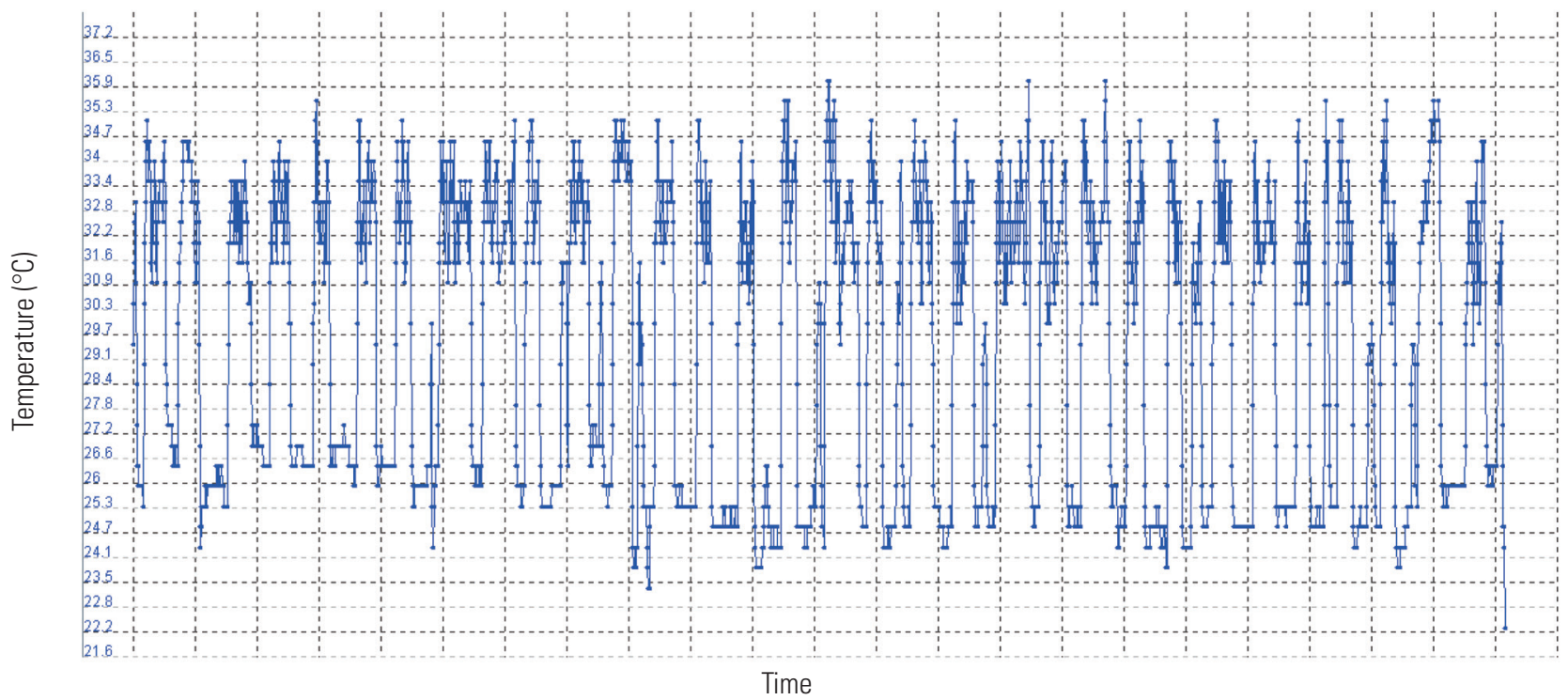

Fig. 2. Recorded temperature using the OneWireViewer software. The information for 2 months of wearing the brace. The x-axis demonstrates the time interval of 15 minutes across 2 data points. The $y$-axis shows the temperature. The threshold temperature was wearing the braces was considered to be $>30^{\circ} \mathrm{C}$. 
Table 1. Demographic characteristics of the recruited participants

\begin{tabular}{lccc} 
Characteristic & Mean \pm standard deviation & Min & Max \\
\hline Age $(\mathrm{yr})$ & $12.89 \pm 1.77$ & 10 & 16 \\
Cobb angle $\left(^{\circ}\right)$ & $63.82 \pm 14.08$ & $38^{\text {a) }}$ & 86 \\
\hline Height $(\mathrm{m})$ & $1.59 \pm 0.10$ & 1.45 & 1.82 \\
Weight $(\mathrm{kg})$ & $54.44 \pm 15.55$ & 27 & 84 \\
\hline Body mass index & $22.00 \pm 4.12$ & 15.35 & 27.99 \\
Follow-up time (day) & $27.11 \pm 10.61$ & 16 & 48 \\
\hline
\end{tabular}

${ }^{a}$ This patient had $67^{\circ}$ kyphosis Cobb angle at initiation bracing but was alleviated to $38^{\circ}$ at the time of participation.

Table 2. Comparison of brace compliance between the three measurement methods

\begin{tabular}{lcccc} 
Compliance rate $(\mathrm{hr})$ & Mean \pm standard deviation & Min & Max & $p$-value \\
Thermochron & $16.00 \pm 4.90$ & 5.79 & 20.29 & $<0.001$ \\
Questionnaire & $19.52 \pm 6.04$ & 9.22 & 23.10 & $<0.001$ \\
Verbal report & $20.21 \pm 6.05$ & 9.22 & 23.00 & $<0.001$ \\
\hline
\end{tabular}

Friedman test indicated that our results were rated differently, $\chi^{2}(2)=26, p$-value $\leq 0.001$.

to determine whether the presence of foam interfered with the sensor and reduced its accuracy, the temperature logger's performance was tested in several situations. The data collection was performed under conditions wherein the foam interval thickness was $2 \mathrm{~mm}, 3 \mathrm{~mm}$, and $5 \mathrm{~mm}$ and was in direct contact with the skin in the brace simultaneously. The results of all of these experiments showed that the temperature differences were $<1^{\circ} \mathrm{C}$.

The only manner in which the brace wearing temperature could have been disturbed during the temperature measuring process (and consequently, affected the results) was if they patient did not wear the brace and left it in an outdoor area. In this case, if the ambient temperature fell to $30^{\circ} \mathrm{C}-37^{\circ} \mathrm{C}$, there was a possibility of an error in the analysis of the algorithm recorded by the temperature logger. In order to control for this potential confounder, each patient was asked (verbally) whether he/she left the brace outdoors or in a car box at any time. The patient and his/ her family did not know about the sensor and the reason for the question; therefore, the probability of bias was minimal.

The third method for assessing patient compliance for the brace-wearing schedule was verbal questioning. For this, the therapist asked the parents or caregiver(s) about the average brace wearing time. The question was framed as follows: "On average, how many hours did your child wear the brace daily and nightly since your last visit?" The score obtained from this question was recorded and evaluated along with the information obtained from the other subjective and objective methods.

\section{Statistical analyses}

The statistical analyses were conducted using IBM SPSS Statistics for Windows ver. 21.0 (IBM Corp., Armonk, NY, USA). Data distribution was assessed for normality using the Shapiro-Wilk test. In order to compare the mean scores obtained from the thermal logger and the subjective compliance measurement methods, the Friedman test was used. To determine the relationships of the compliance rate with the patient's sex, age, and body mass index (BMI), Spearman correlation coefficient analysis was used. The significance level was set at 0.05 for all the tests.

\section{Results}

Of the 21 participants who fulfilled the inclusion criteria, three were excluded because they refused their referrals for continued treatment. Finally, 18 patients (seven boys and 11 girls) completed the study. The mean subject age at the time of the study was $12.89 \pm 1.77$ years, the mean kyphosis Cobb angle was $63.82^{\circ} \pm 14.08^{\circ}$, and the mean duration for follow-up for compliance rate measurement was $27.11 \pm 10.61$ days. Table 1 shows the clinical and demographic characteristics of the study participants. Table 2 shows the comparisons for brace compliance among the 
Table 3. Correlation between the age and body mass index with objective brace compliance

\begin{tabular}{lcc} 
Objective compliance & Age & Body mass index \\
$r$ & 0.794 & 0.907 \\
$p$-value & -0.066 & -0.033 \\
\hline
\end{tabular}

three measurements methods used in the study as per the Friedman statistical test. The results of this test indicated a statistically significant difference in the compliance rates across the three methods of measurements (sensor, questionnaire, and verbal questions). The present results showed no significant correlations of age and BMI with brace compliance (Table 3).

\section{Discussion}

The present results showed a significant difference between the average brace compliances measured using the objective and subjective methods. The results showed a greater compliance based on the parental subjective reports than that measured objectively using the temperature logger. In other words, the brace compliance rate measured using the subjective method was higher than that using the objective method. When considering the proven effects of brace compliance on treatment outcomes, the reported difference may indicate a limitation of the reports on the efficacy of orthotic treatment. This point has been raised by Rahman et al. [22].

We compared a precise questionnaire method that is used more commonly in scientific research with verbal questioning of the patient's parents or guardian(s) to determine the patient's average daily use of the brace, which has been used more commonly in clinical evaluations. The comparison result was interesting because it showed a significant difference between the two methods. This finding strengthens the suggestion that a high risk of error is associated with the use of the routine clinical method for evaluating brace compliance. Moreover, based on the study findings, even the most precise subjective methods are significantly different from the objective ones. The patient and their parents were unaware of the existence of a sensor in the brace. We believe that this is the strength of our study because in most previous studies that have used a sensor, the patient knew about its presence, and this may have interfered with true compliance and resulted in biased results $[23,24]$. In a study Miller et al. [23], the patient's knowledge of the sensor's presence in his or her brace was introduced as a factor that enhanced patient compliance. Lou et al. [24] described the patient's knowledge of the presence of the sensor as a potential contributing factor to the study.

In order to identify the potential factors that affect brace compliance, the effects of each variable, including sex, age, and BMI, were assessed with regard to the compliance rate. Despite the results of the study conducted by Hasler et al. [21], the present study found no correlation between the patient's age and brace compliance. Moreover, sex and BMI were not significantly correlated with brace compliance. These findings suggest that the reasons for compliance and noncompliance of brace use may not be limited to the physical factors of each individual. Future research can focus on the mental perceptions of the patient and the parents with regard to the deformity, determining which has a greater effect on the brace wearing schedule. Moreover, the fear of surgery can also play an important role in patient compliance [25].

Several studies have attempted to determine whether the Milwaukee brace is the most effective orthosis available for the treatment of scoliosis and hyperkyphosis; however, many new orthoses have been designed for treating spinal deformities because of the reported patient rejection of the Milwaukee brace, especially due to the neck component. In the current study, the mean compliance rate for the Milwaukee brace over 1 day and 1 night (23 hours required) was about 16 hours or $70 \%$, objectively. This compliance rate is consistent with that reported by Takemitsu et al. [26] who measured the compliance of the Wilmington brace as well as the findings of Nicholson et al. [11] and Miller et al. [23] who measured the compliance rates of custom-made thoracolumbosacral orthoses for the treatment of spinal deformities in adolescents. However, the studies that aimed to measure the compliance rates of new designs have shown that the same restrictions apply in terms of compliance, and in some cases, the compliance rates of the newer designs were less than those reported in the present study [21]. Therefore, the results of these studies suggest that the factors affecting patient compliance are not limited only to the appearance and nature of the brace.

When considering the results based on the sensor data, the patients were more compliant during the night (bedtime) than during the day. These results correspond to those reported by Nicholson et al. [11]. The present 
study had certain limitations, such as the limited number of subjects, short follow-up duration, and the inclusion of patients with different spinal curve types and different treatment initiation time, even though each patient was only compared with himself/herself. Moreover, the participants and/or their guardians were aware of the information in their questionnaire while answering the verbal report question. Although the number was the same only for one participant, this needs to be considered as a study limitation.

\section{Conclusions}

The present results showed a significant difference between the actual and prescribed brace wearing time in adolescents with Scheuermann's kyphosis. The braces were worn less often than reported by the patients and/or their parents. Therefore, objective compliance assessments of brace use by adolescents with Scheuermann's kyphosis are warranted in the future.

\section{Conflict of Interest}

No potential conflict of interest relevant to this article was reported.

\section{Acknowledgments}

This study was supported by the research committee of Iran University of Medical Sciences.

\section{Author Contributions}

Conception and design: PS, MK, TB, MSG; data acquisition: PS; analysis of data and drafting of the manuscript: PS, MK, TB, MSG; critical revision and final approval of the version to be published: PS, MK, TB, MSG; and supervision: MK, TB, MSG.

\section{References}

1. Fon GT, Pitt MJ, Thies AC Jr. Thoracic kyphosis: range in normal subjects. AJR Am J Roentgenol 1980;134:979-83.

2. Katz DE. Orthoses for spinal deformities. In: Hsu JD, Michael JW, Fisk JR, editors. AAOS atlas of orthoses and assistive devices. Philadelphia (PA): Elsevier
Health Sciences; 2008. p.125-39.

3. Bezalel T, Kalichman L. Improvement of clinical and radiographical presentation of Scheuermann disease after Schroth therapy treatment. J Bodyw Mov Ther 2015;19:232-7.

4. Hart ES, Merlin G, Harisiades J, Grottkau BE. Scheuermann's thoracic kyphosis in the adolescent patient. Orthop Nurs 2010;29:365-71.

5. Korovessis P, Zacharatos S, Koureas G, Megas P. Comparative multifactorial analysis of the effects of idiopathic adolescent scoliosis and Scheuermann kyphosis on the self-perceived health status of adolescents treated with brace. Eur Spine J 2007;16:537-46.

6. Montgomery SP, Erwin WE. Scheuermann's kyphosis: long-term results of Milwaukee braces treatment. Spine (Phila Pa 1976) 1981;6:5-8.

7. Weiss HR, Turnbull D, Bohr S. Brace treatment for patients with Scheuermann's disease: a review of the literature and first experiences with a new brace design. Scoliosis 2009;4:22.

8. Lindeman M, Behm K. Cognitive strategies and selfesteem as predictors of brace-wear noncompliance in patients with idiopathic scoliosis and kyphosis. J Pediatr Orthop 1999;19:493-9.

9. Climent JM, Sanchez J. Impact of the type of brace on the quality of life of adolescents with spine deformities. Spine (Phila Pa 1976) 1999;24:1903-8.

10. Lou E, Hill D, Hedden D, Mahood J, Moreau M, Raso J. An objective measurement of brace usage for the treatment of adolescent idiopathic scoliosis. Med Eng Phys 2011;33:290-4.

11. Nicholson GP, Ferguson-Pell MW, Smith K, Edgar M, Morley T. The objective measurement of spinal orthosis use for the treatment of adolescent idiopathic scoliosis. Spine (Phila Pa 1976) 2003;28:2243-50.

12. Weiss HR, Kleban A. Development of CAD/CAM based brace models for the treatment of patients with scoliosis: classification based approach versus finite element modelling. Asian Spine J 2015;9:661-7.

13. Noonan KJ, Weinstein SL, Jacobson WC, Dolan LA. Use of the Milwaukee brace for progressive idiopathic scoliosis. J Bone Joint Surg Am 1996;78:557-67.

14. Gurnham RB. Adolescent compliance with spinal brace wear. Orthop Nurs 1983;2:13-7.

15. Morton A, Riddle R, Buchanan R, Katz D, Birch J. Accuracy in the prediction and estimation of adherence to bracewear before and during treatment of 
adolescent idiopathic scoliosis. J Pediatr Orthop 2008;28:336-41.

16. Rahman T, Borkhuu B, Littleton AG, et al. Electronic monitoring of scoliosis brace wear compliance. J Child Orthop 2010;4:343-7.

17. Helfenstein A, Lankes M, Ohlert K, et al. The objective determination of compliance in treatment of adolescent idiopathic scoliosis with spinal orthoses. Spine (Phila Pa 1976) 2006;31:339-44.

18. Havey R, Gavin T, Patwardhan A, et al. A reliable and accurate method for measuring orthosis wearing time. Spine (Phila Pa 1976) 2002;27:211-4.

19. Benish BM, Smith KJ, Schwartz MH. Validation of a miniature thermochron for monitoring thoracolumbosacral orthosis wear time. Spine (Phila Pa 1976) 2012;37:309-15.

20. Brox JI, Lange JE, Gunderson RB, Steen H. Good brace compliance reduced curve progression and surgical rates in patients with idiopathic scoliosis. Eur Spine J 2012;21:1957-63.

21. Hasler CC, Wietlisbach S, Buchler P. Objective compliance of adolescent girls with idiopathic sco- liosis in a dynamic SpineCor brace. J Child Orthop 2010;4:211-8.

22. Rahman T, Bowen JR, Takemitsu M, Scott C. The association between brace compliance and outcome for patients with idiopathic scoliosis. J Pediatr Orthop 2005;25:420-2.

23. Miller DJ, Franzone JM, Matsumoto H, et al. Electronic monitoring improves brace-wearing compliance in patients with adolescent idiopathic scoliosis: a randomized clinical trial. Spine (Phila Pa 1976) 2012;37:717-21.

24. Lou E, Raso JV, Hill DL, Mahood JK, Moreau MJ. Correlation between quantity and quality of orthosis wear and treatment outcomes in adolescent idiopathic scoliosis. Prosthet Orthot Int 2004;28:49-54.

25. Brigham EM, Armstrong DG. Motivations for compliance with bracing in adolescent idiopathic scoliosis. Spine Deform 2017;5:46-51.

26. Takemitsu M, Bowen JR, Rahman T, Glutting JJ, Scott CB. Compliance monitoring of brace treatment for patients with idiopathic scoliosis. Spine (Phila Pa 1976) 2004;29:2070-4. 